\title{
Trace Data from Student Solutions to Genetics Problems Reveals Variance in the Processes Related to Different Course Outcomes
}

\author{
Melanie Peffer \\ Molecular, Cellular, and \\ Developmental Biology \\ University of Colorado Boulder \\ Boulder, Colorado \\ melanie.peffer@colorado.edu
}

\author{
David Quigley \\ Institute of Cognitive Science \\ University of Colorado Boulder \\ Boulder, Colorado \\ david.quigley@colorado.edu
}

\author{
Liza Brusman \\ Molecular, Cellular, and \\ Developmental Biology \\ University of Colorado Boulder \\ Boulder, Colorado \\ liza.brusman@colorado.edu
}

\author{
Jennifer Avena \\ Molecular, Cellular, and \\ Developmental Biology \\ University of Colorado Boulder \\ Boulder, Colorado \\ jennifer.avena@colorado.edu
}

\author{
Jennifer Knight \\ Molecular, Cellular, and \\ Developmental Biology \\ University of Colorado Boulder \\ Boulder, Colorado \\ jennifer.knight@colorado.edu
}

\begin{abstract}
Problem solving, particularly in disciplines such as genetics, is an essential but difficult competency for students to master. Prior work indicated that trace data can be leveraged to measure the invisible cognitive processes that undergird learning activities such as problem solving. Building on prior work and given the importance and difficulties associated with genetics problem solving, we used unsupervised statistical methods ( $\mathrm{k}$-means clustering and feature selection) to characterize the patterns of processes students use during genetics problem solving and the relationship to proximal and distal outcomes. At the level of the individual problem, we found that conclusion processes, such as making claims and eliminating possible solutions, was an important interim step and associated with getting a particular problem correct. Surprisingly, we noted that a different set of processes was associated with course outcomes. Students who performed multiple metacognitive steps (e.g. monitoring, checking, planning) in a row or who engaged in execution steps (e.g. using information, drawing a picture, restating the process) as part of problem solving during the semester performed better on final assessments. We found a third set of practices, making consecutive conclusion processes, metacognitive processes preceding reasoning and reasoning preceding conclusions to be important for success at both the problem level and on final assessments. This suggests that different problem-solving processes are associated with success on different course benchmarks. This work raises provocative questions regarding best practices for teaching problem solving in genetics classrooms.
\end{abstract}

Permission to make digital or hard copies of all or part of this work for personal or classroom use is granted without fee provided that copies are not made or distributed for profit or commercial advantage and that copies bear this notice and the full citation on the first page. Copyrights for components of this work owned by others than the author(s) must be honored. Abstracting with credit is permitted. To copy otherwise, or republish, to post on servers or to redistribute to lists, requires prior specific permission and/or a fee. Request permissions from permissions@acm.org.

LAK '20, March 23-27, 2020, Frankfurt, Germany

(c) 2020 Copyright held by the owner/author(s). Publication rights licensed to ACM. ACM ISBN 978-1-4503-7712-6/20/03...\$15.00

https://doi.org/10.1145/3375462.3375503

\section{CCS CONCEPTS}

- Computing methodologies $\rightarrow$ Cluster analysis; Feature selection; • Applied computing $\rightarrow$ Computer-assisted instruction.

\section{KEYWORDS}

Clustering, Problem solving, Genetics, Metacognition

ACM Reference Format:

Melanie Peffer, David Quigley, Liza Brusman, Jennifer Avena, and Jennifer Knight. 2020. Trace Data from Student Solutions to Genetics Problems Reveals Variance in the Processes Related to Different Course Outcomes . In Proceedings of the 10th International Conference on Learning Analytics and Knowledge (LAK '20), March 23-27, 2020, Frankfurt, Germany. ACM, New York, NY, USA, 6 pages. https://doi.org/10.1145/3375462.3375503

\section{INTRODUCTION}

\subsection{Problem Solving in Genetics}

Problem solving involves solving an unstructured problem and is an essential competency for students to master [7]. Although important, problem solving remains challenging for students. Some students have insufficient content knowledge and thus struggle in solving problems unless key pieces of content are provided [2], and other students are unable to appropriately apply their content knowledge [2, 22]. Furthermore, some students fail to use procedural processes used by experts, such as identifying the main concept of a problem [19] or checking one's work [21].

One discipline that relies heavily on problem solving is genetics. In the 21st century, a working knowledge of genetics is critical as members of society are forced to grapple with a wide range of bioethical and moral issues including genetically modified organisms, reproductive technologies, and at-home genetic testing services. Solving problems such as those involving probability and inheritance are no longer limited to biology classrooms but are now relevant to everyone as individuals use consumer genetic testing to ascertain their genetic sequence or genotype data.

Genetics problem-solving is likely to be difficult for students for several reasons. For example, students are required to reason across 
multiple spatial scales, relating processes at a molecular level to physical traits and disease states [13]. Furthermore, genetics problem solving can involve abductive reasoning, in which students begin with an observation and work backward to determine the cause [4]. The mathematical skills required to solve genetics problems (e.g. algebraic modeling, proportional reasoning) also increase the difficulties of these problems [4].

\subsection{Cognitive Processes and Problem Solving}

Solving problems involves cognition occurring at multiple levels, sometimes thought of as a three-level model of processing [12]. The bottom level contains cognitive processes, such as reading and gathering information. The second level involves the metacognitive processes, which include the monitoring of one's cognition. Finally, the third level is epistemic cognition, where one considers what they know and why and how they know it. Said otherwise, metacognition is "thinking about thinking" and epistemic cognition is "knowing about knowing" [9].

In the context of problem solving, metacognition is thought to be a key link between a learner's ability to understand and reflect on how they learn and apply that knowledge to solving new problems [10]. Metacognition is described as containing two components: metacognitive knowledge and metacognitive skills. Metacognitive knowledge includes understanding of how one thinks and how others think about problems, understanding of the task at hand, and strategies for accomplishing a task [25]. Metacognitive skills are processes used by an individual to regulate their own learning and cognition such as through planning, evaluating, and monitoring [25].

Although metacognition is well known to be an important component of both problem solving and learning in general, student metacognition is typically not visible [6]. Therefore, educators and researchers are forced to view metacognitive processes indirectly via student activities in some real-world context, task, or environment. In the context of epistemic cognition, in particular epistemological beliefs about science as situated in authentic science inquiry, prior work has suggested that practices in inquiry reflect epistemological beliefs about the nature of science knowledge [15]. Therefore, trace data from tasks can be used as a proxy for a students' invisible cognitive processes.

\subsection{Trace Data for Understanding Self-Regulated Learning and Problem Solving}

Previous work in the field of learning analytics has used trace data to understand self-regulated learning, which is considered to be both a metacognitive process and also a construct that can be studied to understand metacognition [5]. Self-regulation is also a critical skill for fostering life-long learning and refers to the learner as an active participant in their own learning processes [10]. [23] utilized process mining techniques and sequential information to assess the role of metacognitive prompting on self-regulated learning. The authors found that students in the metacognitive prompting group demonstrated more metacognitive learning activities and had improved transfer performance compared to the control group which did not receive metacognitive prompts. Mediation analysis indicated that metacognitive activities, and monitoring in particular, increased transfer performance. The authors also indicated that examination of the order of process models between the prompting and control group indicated distinct process patterns, which could be pedagogically targeted to improve metacognitive prompting [23]. Other work has used trace data to uncover teacher-level differences in the classroom [18] and used those results to redesign the workflow of their tool to scaffold the activity process [17]. Overall, these works highlight the importance and utility of using trace data with sequential order preserved to detect differences in self-regulated learning processes.

Self-regulated learning and metacognition are also important for problem solving. [10] examined the role of different types of prompts for stimulating reflection during problem solving. In this study, the problem context provided to students was to identify differences between infection by influenza and human immunodeficiency viruses through the creation of a concept map. Students were split into three groups, control (no prompting), generic prompting (general suggestions for problem solving) and direct prompting (specific prompts for particular metacognitive skills such as planning or monitoring). Models were then assessed using the Highly Integrated Model Assessment Technology and Tools or HIMATT which compares student generated models to those of experts for automatic, fast, high throughput assessment. [10] found that the generic prompting group outperformed the other two, demonstrating increased gain in content knowledge and enhanced understanding of the problem scenario. However, the type of problem solving examined by [10] is different from the abductive problems prevalent in genetics classrooms.

\subsection{Current Study}

Since previous work has suggested that learning analytics can be used to generate novel insights about self-regulated learning via trace data $[23,24]$ we investigated whether trace data could also reveal cognitive processes in action during abductive problem solving. Outcomes were examined both at the level of practice problems and end of the semester final assessments, which included the standardized genetics concept assessment (GCA; [20]) and instructor generated final exam (FE).

Our work builds on previous findings within the learning analytics community to specifically look at abductive problem solving in a genetics context. Insights from this study revealed that different problem-solving practices are associated with success at different course benchmarks, including at the level of individual problems and on final assessments. This suggests that different processes are associated with success at the individual problem level compared to performance on final assessments. Since different processes are associated with success at different levels, this could lead to targeted pedagogical intervention to improve not only performance on practice problems, but attainment of course benchmarks. This work also demonstrates how learning analytics can be used to reveal important insights into classroom learning. 


\section{METHODS}

\subsection{Data Collection}

2.1.1 Participants. We analyzed data collected from 295 undergraduate students enrolled in an introductory genetics course. Students who consented to participate in research consisted of $71 \%$ of total students enrolled. The course was mainly first and second year students pursuing biology degrees and approximately $10 \%$ of students were non-STEM majors. Although we did not collect detailed demographic data, the course is traditionally $60 \%$ female. Use of human subjects was approved by the University of Colorado Boulder Institutional Review Board (protocol 16-0511).

2.1.2 Intervention. Students were given the opportunity to complete practice problems for extra credit. Each constructed response question had a single correct answer, and students were asked to explain their thinking process as they solved each problem leading to their final answer. Assignment instructions and question details can be found in [2].

Students completed each set of problems using the online survey platform Qualtrics, for which they could earn $2 / 3$ of the extra credit points for writing out their process and $1 / 3$ for providing a correct answer. The first set of problems consisted of three isomorphic questions for each of three content areas: calculating the probability of inheritance using pedigree information ("probability"), predicting the cause of an extra chromosome in a gamete ("nondisjunction"), and interpreting both a DNA gel and pedigree to identify the mode of inheritance of a trait ("gel/pedigree"). Each student was randomly assigned to two of three content areas. The second problem set included three isomorphic questions that all students were provided about predicting the probability of inheritance of linked genes "recombination." Questions were presented in a random order within each content area. Students had the opportunity to complete up to nine questions.

The final exam for the course contained items from the Genetics Concept Assessment (GCA; [20]) and instructor generated final exam items (FE). The GCA is a 25 -item multiple choice assessment developed to measure conceptual understanding of key genetics topics. It is given both pre- and post- course to demonstrate learning gains as a result of instruction [20]. Thus, the GCA is included on the final exam for this course, and comprises 50 out of 150 possible points; the rest of the questions on the final exam were a set of multiple-choice questions that address content not on the GCA (25 points) and constructed response questions that address content integrated across all topics in the course ( 75 points). Since one is a standardized assessment of genetics knowledge and the other is course-specific, we included each as a different outcome metric in our analysis.

\subsection{Analysis}

2.2.1 Coding. To quantify the problem-solving processes written by the students, we used an emergent coding approach that drew from categories established by [16] and extended in [3] to identify 19 different processes that fall into five broad categories. Table 1 shows the subcategories used for this study with their associated categories. Four raters iteratively established this scheme, reaching a final interrater agreement of $85 \%$. Each statement within each
Table 1: Process Categories

\begin{tabular}{|l|l|}
\hline Category & Subcategories \\
\hline Conclusion & Eliminating, Claiming \\
\hline Metacognition & Monitioring, Checking, Planning \\
\hline Orientation & $\begin{array}{l}\text { Clarifying, Recalling Information, Identify- } \\
\text { ing Similarities Between Question Types }\end{array}$ \\
\hline Reasoning & $\begin{array}{l}\text { Justifying Answers with Evidence, Reason- } \\
\text { ing }\end{array}$ \\
\hline Execution & $\begin{array}{l}\text { Using Information, Calculating, Creating a } \\
\text { Visual Representation, Restating the Pro- } \\
\text { cess }\end{array}$ \\
\hline
\end{tabular}

student response was given a code, preserving process order, using HyperRESEARCH software [1]. Each answer was also coded as a binary variable (correct or incorrect). Performance on the GCA and Final Exam are represented as a percent of 100.

2.2.2 Feature Transformation and Selection Analysis. Each process performed by students was coded according to the process categories (Table 1). The broad categories were used to generate strings of processes, similar to $[14,18]$. For example, let's say a student was working on a probability problem. They began by recalling information about probability from class, then clarifying the part of the scenario with data relevant to answer. Then they calculated the resulting probability, checked that their answer fit within the expectation, and stated their final claim. This sequence of actions was captured as [O, O, E, M, C].

From these sequences for each problem solved by each student, We built counts of all possible action bigrams in order to capture the sequential nature of these problem-solving descriptions. In our example above, these bigrams would be [OO, OE, EM, MC]. These bigrams were fed into a Greedy Stepwise attribute selection process using Weka [8]. This analysis generated a selection of the most informative features for each problem type in predicting outcomes on the practice problem at hand, the GCA score, and the FE score.

2.2.3 Cluster Analysis. K-means clustering was performed using WEKA [8]. For this exploratory analysis, we built clusters using the bi-gram features from the full dataset and found the optimal number of clusters (k) using the elbow method [11]. Briefly, the sum of squared errors (SSE) was calculated for each $\mathrm{k}$ and the ideal number of clusters was chosen by determining the $\mathrm{k}$ for which the SSE is low and increasing $\mathrm{k}$ does not significantly decrease SSE. These cluster assignments were then recombined to evaluate the distribution of problem-level and course-level outcome scores among the unsupervised clusters. For comparisons between clusters on the numeric GCA and FE scores, we used pairwise t-tests. To compare between clusters on the binary problem correctness value, we used pairwise z-tests of the probability of correctness within the cluster.

\section{RESULTS}

Our analyses demonstrated which bigram features are important in certain clusters (Table 2), along with which bigrams are predictive of certain outcome measures (Table 3 ). 
Table 2: Cluster Centroids

\begin{tabular}{|c|c|c|c|}
\hline Feature & $\begin{array}{l}\text { Cluster } 1 \\
\text { Average }\end{array}$ & $\begin{array}{l}\text { Cluster } 2 \\
\text { Average }\end{array}$ & $\begin{array}{c}\text { Cluster } 3 \\
\text { Average }\end{array}$ \\
\hline $\mathrm{CC}$ & 0.12 & 0.12 & 0.02 \\
\hline $\mathrm{CM}$ & 0.26 & 0.03 & 0.02 \\
\hline $\mathrm{CO}$ & 0.07 & 0.03 & 0.01 \\
\hline $\mathrm{CR}$ & 0.44 & 0.63 & 0.14 \\
\hline $\mathrm{CE}$ & 0.14 & 0.22 & 0.07 \\
\hline $\mathrm{MC}$ & 0.11 & 0.05 & 0.00 \\
\hline MM & 0.09 & 0.02 & 0.01 \\
\hline $\mathrm{MO}$ & 1.41 & 0.14 & 0.05 \\
\hline MR & 0.15 & 0.04 & 0.02 \\
\hline $\mathrm{ME}$ & 0.62 & 0.06 & 0.15 \\
\hline $\mathrm{OC}$ & 0.35 & 0.16 & 0.01 \\
\hline $\mathrm{OM}$ & 0.87 & 0.10 & 0.09 \\
\hline $\mathrm{OO}$ & 1.2 & 0.30 & 0.39 \\
\hline OR & 0.31 & 0.19 & 0.10 \\
\hline $\mathrm{OE}$ & 0.89 & 0.35 & 0.70 \\
\hline $\mathrm{RC}$ & 0.45 & 1.04 & 0.04 \\
\hline $\mathrm{RM}$ & 0.28 & 0.04 & 0.01 \\
\hline $\mathrm{RO}$ & 0.06 & 0.03 & 0.04 \\
\hline RR & 0.09 & 0.12 & 0.07 \\
\hline $\mathrm{RE}$ & 0.85 & 0.29 & 1.28 \\
\hline $\mathrm{EC}$ & 0.56 & 0.11 & 1.02 \\
\hline EM & 0.79 & 0.06 & 0.07 \\
\hline $\mathrm{EO}$ & 0.30 & 0.12 & 0.21 \\
\hline ER & 0.84 & 0.65 & 1.15 \\
\hline $\mathrm{EE}$ & 1.13 & 0.54 & 1.30 \\
\hline Count & 159 & 643 & 712 \\
\hline $\begin{array}{c}\text { Probability of Problem } \\
\text { Correctness }\end{array}$ & 0.67 & 0.64 & 0.56 \\
\hline Average Score on GCA & 76.62 & 73.34 & 73.59 \\
\hline Average Score on FE & 75.10 & 72.41 & 72.59 \\
\hline
\end{tabular}

\subsection{Bigram Clustering Reveals Differences in Processes and Probability of Answering Problems Correctly}

Our k-means cluster analysis resulted in an optimal size of 3 clusters. The centroids for each feature and the distribution of outcome variables can be found in Table 2 . In brief, we note that Cluster 1 was the smallest and unique in the number of conclusion - metacognition (CM), metacognition - orientation (MO), metacognition - execution (ME), orientation - metacognition $(\mathrm{OM})$, orientation - execution $(\mathrm{OE})$, reasoning - metacognition (RM), reasoning - execution (RE), and execution - metacognition (EM) bigrams. Notably, The probability of getting the current problem correct is significantly lower for students in Cluster 3 when compared to the other two clusters (Cluster 1 to Cluster 2: $\mathrm{Z}=0.65, \mathrm{p}=0.52$; Cluster 1 to Cluster $3: \mathrm{Z}=$ $2.55, \mathrm{p}=0.01$; Cluster 2 to Cluster $3: \mathrm{Z}=3.11, \mathrm{p}=0.00$ ). In addition, Cluster 3 demonstrated much higher numbers of reasoning - execution (RE) statements, execution-conclusion (EC) compared to the other two clusters. Cluster 2 was similar to Cluster 1 in terms of
Table 3: Bigram Feature Selection by Outcome

\begin{tabular}{|c|c|c|c|}
\hline $\begin{array}{l}\text { Feature } \\
\text { Bigram }\end{array}$ & $\begin{array}{l}\text { Primary: } \\
\text { Practice } \\
\text { Problem } \\
\text { Correctness }\end{array}$ & $\begin{array}{l}\text { Primary: } \\
\text { GCA Score }\end{array}$ & $\begin{array}{c}\text { Primary: FE } \\
\text { Score }\end{array}$ \\
\hline $\mathrm{CC}$ & $\mathrm{X}$ & $\mathrm{X}$ & $\mathrm{X}$ \\
\hline $\mathrm{CM}$ & $\bar{X}$ & & \\
\hline $\mathrm{CO}$ & $\mathrm{X}$ & & \\
\hline $\mathrm{CR}$ & $\bar{X}$ & & \\
\hline $\mathrm{CE}$ & $\mathrm{X}$ & & \\
\hline $\mathrm{MC}$ & $\mathrm{X}$ & & \\
\hline MM & & $\mathrm{X}$ & $\mathrm{X}$ \\
\hline $\mathrm{MO}$ & & & \\
\hline MR & $\mathrm{X}$ & $\mathrm{X}$ & $\mathrm{X}$ \\
\hline $\mathrm{ME}$ & & & $\mathrm{X}$ \\
\hline $\mathrm{OC}$ & & & \\
\hline $\mathrm{OM}$ & $\mathrm{X}$ & & $\mathrm{X}$ \\
\hline $\mathrm{OO}$ & & & \\
\hline OR & $\mathrm{X}$ & & \\
\hline $\mathrm{OE}$ & & & \\
\hline $\mathrm{RC}$ & $\mathrm{X}$ & $\mathrm{X}$ & $\mathrm{X}$ \\
\hline $\mathrm{RM}$ & & & \\
\hline $\mathrm{RO}$ & $\mathrm{X}$ & & $\mathrm{X}$ \\
\hline RR & $\mathrm{X}$ & & \\
\hline $\mathrm{RE}$ & & & \\
\hline EC & & & \\
\hline EM & & $\mathrm{X}$ & \\
\hline EO & & $\mathrm{X}$ & $\mathrm{X}$ \\
\hline ER & & $\mathrm{X}$ & $\mathrm{X}$ \\
\hline $\mathrm{EE}$ & & & $\bar{X}$ \\
\hline
\end{tabular}

probability of getting the individual problem in question correct but differed from the other clusters in terms of increased reasoning - conclusion (RC) processes and lower execution - execution (EE) processes than the other two clusters.

\subsection{Feature Selection Demonstrates Different Bigrams Associated with Success at Problem Solving and Overall Course Outcomes.}

We next examined which bigrams were most important at predicting outcomes, both at the level of the individual problem and overall mastery with course material as shown by GCA and FE scores. Our feature selection results are presented in Table 3. Xs denote features that were found to be principle components for predicting performance on a particular outcome measure. When considering only the possibility of a correct answer on the problem at hand, we found the features marked in Table 3, column 2 to be principle components for predicting correctness. When considering outcome mastery for the course, namely the GCA and FE scores, columns 3 and 4 respectively represent the principle components for predicting higher scores. 


\section{DISCUSSION}

\subsection{Bigram Clustering: Potential Insights for Pedagogical Intervention}

Our bigram model revealed distinct distributions of students' sequences of thinking. The only significant difference in outcomes was the lower proportion of correct answers on practice problems in Cluster 3. In reviewing the features that informed Cluster 3, they show lower or comparable centroids for all features except reasoning followed by execution (RE), execution followed either by conclusions or reasoning (EC and ER), and orienting followed by execution (OE) only when compared to Cluster 2 . We also noted that Cluster 3 has an underrepresentation of bigrams including metacognitive processes. Since Cluster 3 contains students who are statistically less likely to get an individual practice problem correct, and metacognition is important for problem solving [10], it may not be surprising that Cluster 3 contains students with less metacognitive processes who also performed poorer on practice problems.

However, our observations concerning the prevalence of execution steps in the absence of metacognition is notable. Execution steps involve concrete actions such as using information provided in the problem or drawing from one's own knowledge, performing a mathematical calculation, or making a representation to help solve the problem, such as generating a Punnett Square or pedigree chart. Students' focus on "doing" may come at the expense of metacognitive tasks. Alternatively, becoming skilled at solving genetics problems may involve a shift to incorporate metacognitive tasks alongside cognitive tasks. Since instruction on using metacognition as part of task is important for students to incorporate metacognition into their work [24], this work undergirds the importance of modeling metacognitive strategies alongside cognitive strategies for solving genetics problems.

\subsection{Different Features are Associated with Distinct Outcomes}

Our most noteworthy finding was that there are three sets of sequence features: those that predict correctness on individual practice problems, those that predict mastery of course content, and those that are associated with both. Since one might expect that success in solving genetics problems, such as the practice problems analyzed here, would be reflected on both final assessments, it is somewhat surprising that there are distinct processes associated with success on each. One possible interpretation is that the conclusions statements are important for specific problems, but additional processes are important for understanding student performance on different kinds of questions, such as the multiple-choice questions on the GCA and short answer questions on the FE.

Specific only to practice problem-level performance was the generation of interim conclusions, as demonstrated by conclusion actions followed by non-conclusion actions (CM, CO, CR and CE). This indicates that a successful strategy is drawing interim conclusions while problem-solving. It is also possible that the overabundance of conclusion statements could be an artifact from data collection since students were asked to describe their process in reaching a conclusion. However, these processes did not correlate with overall performance on final assessments, even though these assessments contained some similar types of problems. Instead, we observed that sequential metacognition steps (MM) and an execution process followed by either a metacognitive (EM), orienting (EO), reasoning (ER), or another execution (EE) step were associated with better performance on the GCA and the FE. Connecting this finding to our bigram clustering in Table 2, we note that execution followed by reasoning (ER) sequences are more highly represented in Cluster 3. Cluster 3 was not different from the other clusters in terms of overall score on the GCA or FE, but was with regards to probability of getting a practice problem correct. This suggests that execution processes, particularly execution processes followed by reasoning processes are important for performance on final assessments, but not on practice problems.

We also observed that some processes were associated with mastery at both levels, namely sequential conclusion statements (CC) metacognitive processes preceding reasoning statements (MR), reasoning followed by conclusions (RC), and to a lesser extent, orienting followed by metacognition (OM) and reasoning following by orienting (RO). Within the context of problem solving, it makes sense that students who, for example, use metacognition prior to reasoning, or have reasoning statements leading to conclusions would be better at solving both individual problems and have higher final assessment scores. Although execution steps were associated with being less likely to answer individual practice problems correctly, such steps were not associated with final assessment outcomes. This lends support to the conclusion that execution steps, while obviously required for solving a problem, may be less important for subject mastery than other supporting processes. Students commonly focus on practicing execution steps, such as generating Punnett Squares. However, if they have a limited understanding of why they are using this representation, or how to gather information from it, they may be less successful in learning genetics.

Finally, it is important to note the features that were not predictive for either set of outcomes. We noted that metacognition followed by orienting (MO), orienting followed by concluding or execution (OC and $\mathrm{OE}$ ), reasoning followed by metacognition or execution (RM and RE), and execution followed by concluding (EC) were not significant to success on either practice problems or final assessments. Some bigrams, such as orienting followed by conclusion (OC) make sense to be unimportant, because this could be construed as unintuitive or counterproductive to problem solving. Other bigrams we would expect to be important for predicting success on either outcome measure. For example, metacognition followed by orienting (MO) could be expected in successful problem solving. In a hypothetical scenario, it could be possible for a student to check their current trajectory and recognize that their current strategy is not working. Once this realization is made, the student would then re-orient before trying again. These results warrant further investigation, possibly extending from bigrams to trigrams.

\subsection{Limitations}

As mentioned above, one possible limitation of this study is that the abundance of conclusion statements during problem solving may be an artifact of our method for collecting process data. However, since we observed other specific processes as important for other 
course outcomes, this suggests we were still able to capture interesting and novel student choices during problem solving. Another potential limitation is that the process data was self-reported by the students. Although this may have resulted in the loss of some information, during the coding process we were able to compare student statements of processes to their actual work to complete each problem. Future work could re-evaluate these processes in an automated support system that relies on automatically captured clickstream data. As with many educational research papers, we also must consider the chance for self-selection bias, both in regards to participating in research and since the practice problems analyzed here were for extra credit, rather than graded homework.

\subsection{Conclusions}

The results presented here are interesting and unexpected and add to a growing literature underscoring the utility of using learning analytics to better understand learning processes in real-world classrooms. We found that different problem-solving processes were associated with success on different assessments. Although some processes were associated with improved performance on both the individual problems and final assessments, others were strongly associated with one or the other. This leads to several notable next steps for improved pedagogy in genetics classrooms. For example, since either progressive metacognition steps or metacognition steps followed by execution steps were associated with enhanced performance on both the problem itself and final assessments, these may be important to address in the classroom. The importance of this paper is not only that it provided novel and valuable insights into genetics problem solving, but potential avenues for learning analytics informed pedagogical interventions.

\section{ACKNOWLEDGMENTS}

This work was supported by the National Science Foundation (DUE 1711348). We thank Oscar Whitney for assistance with initial question development, testing and process coding and Betsy McIntosh for process coding. We also thank Daniel Weaver for his assistance processing the data from HyperRESEARCH into strings for analysis in WEKA.

\section{REFERENCES}

[1] HyperRESEARCH 3.7.3. 2015. Researcherware, Inc. http://www.researchware. com.

[2] Jennifer S. Avena and Jennifer K. Knight. 2019. Problem Solving in Genetics: Content Hints Can Help. CBE-Life Sciences Education 18, 2 (2019), ar23. https: //doi.org/10.1187/cbe.18-06-0093 PMID: 31144570.

[3] Jennifer S Avena, Betsy B McIntosh, Whitney Oscar, Ashton Wiens, and Jennifer K Knight. [n.d.]. Successfully solving genetics problems requires reasoning. $C B E-$ Life Sciences Education ([n.d.]).

[4] Albert Corbett, Linda Kauffman, Ben Maclaren, Angela Wagner, and Elizabeth Jones. 2010. A Cognitive Tutor for genetics problem solving: Learning gains and student modeling. Fournal of Educational Computing Research 42, 2 (2010), 219-239.

[5] Eva Durall and Begoña Gros. 2014. Learning Analytics as a Metacognitive Tool.. In CSEDU (1). 380-384.

[6] Andrew Gibson, Kirsty Kitto, and Peter Bruza. 2016. Towards the discovery of learner metacognition from reflective writing. Journal of Learning Analytics 3, 2 (2016), 22-36

[7] Lila Gleitman, Anna Papafragou, Keith J Holyoak, and Robert G Morrison. 2005 Cambridge handbook of thinking and reasoning. (2005).

[8] Mark Hall, Eibe Frank, Geoffrey Holmes, Bernhard Pfahringer, Peter Reutemann, and Ian H Witten. 2009. The WEKA data mining software: an update. ACM SIGKDD explorations newsletter 11, 1 (2009), 10-18.
[9] Barbara K Hofer. 2004. Epistemological understanding as a metacognitive process: Thinking aloud during online searching. Educational Psychologist 39, 1 (2004), 43-55.

[10] Dirk Ifenthaler. 2012. Determining the effectiveness of prompts for self-regulated learning in problem-solving scenarios. Fournal of Educational Technology \& Society 15, 1 (2012), 38-52.

[11] David J Ketchen and Christopher L Shook. 1996. The application of cluster analysis in strategic management research: an analysis and critique. Strategic management journal 17, 6 (1996), 441-458.

[12] Karen Strohm Kitchner. 1983. Cognition, metacognition, and epistemic cognition. Human development 26, 4 (1983), 222-232.

[13] Teresa L McElhinny, Michael J Dougherty, Bethany V Bowling, and Julie C Libarkin. 2014. The status of genetics curriculum in higher education in the United States: goals and assessment. Science \& Education 23, 2 (2014), 445-464.

[14] Melanie Peffer, David Ouigley, and Mehrgan Mostowfi. 2019. Clustering Analysis Reveals Authentic Science Inquiry Trajectories Among Undergraduates. In Proceedings of the 9th International Conference on Learning Analytics \& Knowledge. ACM, 96-100.

[15] Melanie E Peffer and Niloofar Ramezani. 2019. Assessing epistemological beliefs of experts and novices via practices in authentic science inquiry. International fournal of STEM Education 6, 1 (2019), 3.

[16] Luanna B Prevost and Paula P Lemons. 2016. Step by step: Biology undergraduates' problem-solving procedures during multiple-choice assessment. CBE-Life Sciences Education 15, 4 (2016), ar71.

[17] David Quigley, Conor McNamara, Jonathan Ostwald, and Tamara Sumner. 2017. Using learning analytics to Understand scientific Modeling in the classroom. Frontiers in ICT 4 (2017), 24

[18] David Quigley, Jonathan Ostwald, and Tamara Sumner. 2017. Scientific Modeling: Using Learning Analytics to Examine Student Practices and Classroom Variation. In Proceedings of the Seventh International Learning Analytics \&\#38; Knowledge Conference (LAK '17). ACM, New York, NY, USA, 329-338. https://doi.org/10. $1145 / 3027385.3027420$

[19] Julia I Smith, Elijah D Combs, Paul H Nagami, Valerie M Alto, Henry G Goh, Muryam AA Gourdet, Christina M Hough, Ashley E Nickell, Adrian G Peer, John D Coley, et al. 2013. Development of the biology card sorting task to measure conceptual expertise in biology. CBE-Life Sciences Education 12, 4 (2013), 628-644.

[20] Michelle K Smith, William B Wood, and Jennifer K Knight. 2008. The genetics concept assessment: a new concept inventory for gauging student understanding of genetics. CBE-Life Sciences Education 7, 4 (2008), 422-430.

[21] Mike U Smith. 1988. Successful and unsuccessful problem solving in classical genetic pedigrees. Journal of Research in Science Teaching 25, 6 (1988), 411-433.

[22] Mike U Smith and Ron Good. 1984. Problem solving and classical genetics: Successful versus unsuccessful performance. Journal of Research in Science Teaching 21, 9 (1984), 895-912.

[23] Christoph Sonnenberg and Maria Bannert. 2015. Discovering the effects of metacognitive prompts on the sequential structure of SRL-processes using process mining techniques. Fournal of Learning Analytics 2, 1 (2015), 72-100.

[24] Philip H Winne. 2017. Learning analytics for self-regulated learning. Handbook of learning analytics (2017), 241-249.

[25] Anat Zohar and Sarit Barzilai. 2013. A review of research on metacognition in science education: Current and future directions. Studies in Science education 49 , 2 (2013), 121-169. 